\title{
REPLACING THE USE OF TEXTURE AND SEALED AREA IN URBAN FABRIC CLASSIFICATIONS BY INTEGRATING VOLUME AND OBJECT BASED DISTANCE CALCULATIONS
}

\author{
R. de Kok $^{\mathrm{a},{ }^{*}}$, P. Wezyk ${ }^{\mathrm{b}}$, B. Hejmanowska ${ }^{\mathrm{c}}$, J. Książek $^{\mathrm{c}}$ \\ a ProGea.PL, \\ ${ }^{\mathrm{b}}$ Laboratory of Geomatics, Institute of Forest Resource Management, Faculty of Forestry, University of Agriculture in Krakow, \\ ${ }^{c}$ AGH University of Science and Technology, Kraków, Poland, Faculty of Mine Surveying and Environmental Engineering, \\ Department of Geoinformation, Photogrammetry and Remote Sensing of Environment. \\ roeland_de_kok@hotmail.com
}

KEY WORDS: Urban Fabric, Distance to Neighbours, Perception, Thiessen, GEOBIA

\begin{abstract}
:
The classification of urban fabric can be achieved using the information of individual buildings and measure their distance to all image objects in the map. The distance to a variety of building types return various areas, which can be associated with dense and less dense urban fabric. The resulting classification can be used as an alternative or as a control mechanism on normal urban fabric classification. The method can be used to extend existing manuals on urban fabric.
\end{abstract}

\section{REDEFINING URBAN FABRIC}

\subsection{Urban fabric by proxy}

The Urban fabric is described in terms of "dense", "less dense", "continuous" and "discontinuous". These classifications are usually applied in projects such as Corine and Urban Atlas (EU, 2011). The driving factor for the urban fabric classification is the layer of "soil sealing" and the loosely defined "texture" (Bossard et.al. 2000, EU, 2011). The role of both the Red and the Infrared channel for mapping the vegetation is essential. In remote sensing, the degree of imperviousness can be estimated with proxy parameters that quantify the cover of green vegetation, which en gross can be considered inversely correlated with the degree of surface imperviousness in urban or built-up areas (Gangkofner et. Al, 2010) The surface imperviousness can than play a role in classifying dense and less dense urban fabric.

\subsection{Classifying by building type}

This study introduces a method to classify urban fabric without relying on the availability of the Red and the Infrared spectral channels. Although the manual for Urban Atlas explicitly states that building type should not play a role in urban fabric classification (EU, 2011) it is the intuitive and historically developed human perception on the topographical map that allows to distinguish urban zones related to building type (see also Chapter 2.1). For reasons of perception and the strive for simulating the unique human vision with computer vision (Marr, 1982), the core dataset used in this study is based on data that allows the extraction of the size and height of individual buildings. There are different datasets for retrieving such information; Lidar data that goes back till end of 20th century. Alternatively, classic photogrammetric imagery that relies on parallax (see o.a. Avery and Gradon, 1992). This data has the advantage that it goes back till beginning of 19th century.

\subsection{Extracting groups of single buildings}

The level of detail in cadastral maps deviates from satellite based urban mapping. Details on individual buildings in cadastral GIS are standard. Most in the form of the building footprints. The visualization of the cadastral map only hints at the inherent grouping of neighbouring buildings with similarities in footprint (see chapter 2.1 on perception). Between urban mapping for international use, based on satellite data and the cadastral maps for local use, based on surveying, there are huge differences in the budget, production timeline, users and mapping techniques. The aim is to move the information derived from grouped individual buildings up to the level of 25:000 (trans) national scale, making it comparable to the mapping scale of Corine and Urban Atlas.

After demonstrating initial results, the presented study can function as a basis to discuss the relevance of urban maps from historical data. The results can also become an alternative to overcome the problems related to urban change detection methods. The analysis could further be applied in the near future for a full automatic tagging on existing Urban Atlas polygons that might require an update.

\subsection{The primal sketch to group image parts}

Urban fabric is classified by a proxy parameter. The measurement by proxy does not explain the real physically density of urban area. There is few conceptual information in the manuals on this topic and even no information on the ontology of urban fabric. Although, outside of the manuals, the scientific literature is extensive (o.a. Montenergro, 2009). Starting with a classical figure from the Urban Atlas mapping guide (EU, 2006), it is Fig $1 \mathrm{~A}$. This figure can be related to the very early manual of Corine landcover ( Bossard e.a. , 2000). It is one of the rare illustrations related to the concept of urban fabric. Now any explicit information on this image is just dropped in the 2006 Urban Atlas guide, maybe for good reasons.

\footnotetext{
* Corresponding author
} 


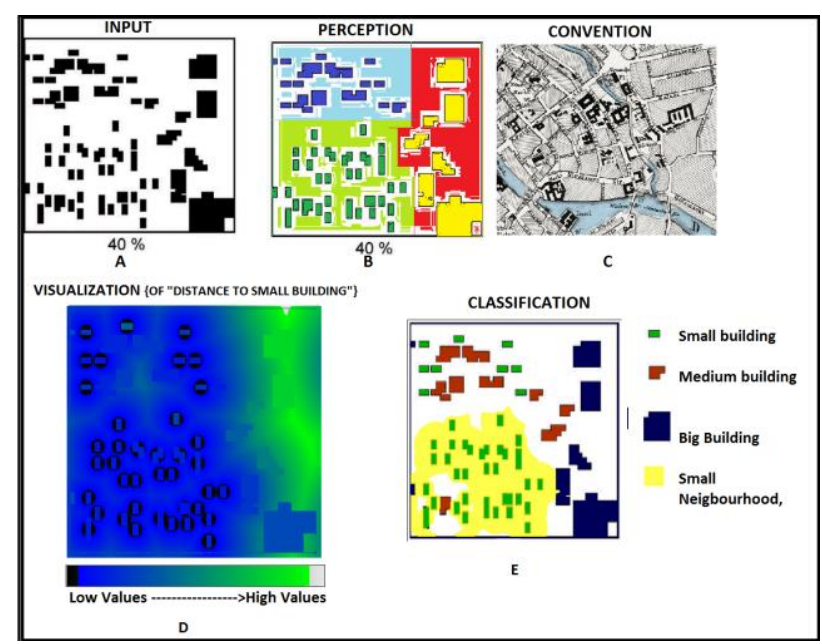

Figure 1A-E. Illustrations on the various concepts of map interpretation. A; Computer Vision Input value 40\%, B; A sketch on Fig 1 A to illustrate the Human Perception, C, Historical Convention on the topographic map around 1850, D; Feature Value Visualization on Fig1A, E; Image Classification.

Although Fig 1 A might seem extremely simple, the perception (Marr, 1982) is quite complicated. First of all, the classical layout of the historical topographic map at large scale depicts urban areas with individual footprints of selected buildings. Fig $1 \mathrm{C}$ is a snapshot of a 19th century map of Alexanderplaz, Berlin. The Fig $1 \mathrm{C}$ is related to cartographic convention on how to display the symbol of a large building with the printing technique of that time. The " $40 \%$ "annotation is a radical break with that convention. No person would ever "see" " $40 \%$ ". This " $40 \%$ " annotation in Fig1A is a computer vision fossil. It only hints at the simplest value at which the image can be processed using the most basic image processing techniques. With a revolution in computer vision, the $40 \%$ must be reduced to only one of the multitude of measurements that makes Fig 1 A accessible to computer processing. So, if a person does not see " $40 \%$ ", than what is the true perception of Fig 1 A ?

Although a more in-depth explanation on perception would surely improve the manuals, a first attempt is achieved using Fig. 1B which is derived from Fig1A in limited accordance with the very extensive theory on perception used by Marr (Marr, 1982). In Fig. 5B a "primal sketch" uses manually added colours to illustrate how a person "sees" the city. Perception in different stages, as described by Marr (1982) involves the areas that are interpreted as belonging to a group or cluster in the image. A naive perception of the urban fabric would separate the Fig $1 \mathrm{~A}$ in different building types and zones. This interpretation process is approached by adding the colours in Fig 1B. In addition to the explanation on the visual interpretation in a modern manual, we are now in urgent need of an extension of the manuals with computer protocols.

\subsection{The process tree}

To illustrate this need for an extension of the manual, the image-object-feature "distance to building" is used. This is an example to explain the need of a process protocol accompanying the illustration of the manual. Except for the initial $40 \%$ in Fig $1 \mathrm{~A}$, more and more complex values can be extracted from the conceptual illustration of Fig. 1A. Fig 1 D shows the distance of all pixels towards the nearest small buildings. The yellow area in Fig 1 E ("Goal1" in the following process tree) is classified with the following process. For the final paper, the protocol will be added here:..

The yellow polygon of Fig 1 E classified by the to be shown protocol only approaches the manually coloured green district of Fig $1 \mathrm{~B}$ and could be associated by proxy as an area of sparse urban fabric with small houses. It is clear that not all small (green) buildings in Fig $1 \mathrm{D}$ are surrounded by a yellow area. The rule set can be expanded and refined for generalization purposes. However, the role of it's essential feature "Distance to Neighbour" remains.

\section{FROM SIMULATION TO REAL DATA}

\subsection{Thiessen area by proxy}

The feature display of Fig $1 \mathrm{D}$ can be applied to real data as well. Fig 2 Shows the study area over Cracow with the castle Wawel along the river Wisla $(20 \times 10 \mathrm{Km})$, where Fig. 3 shows the same feature distance to building in exact area of Fig 2 with the feature as used in Fig 1D.

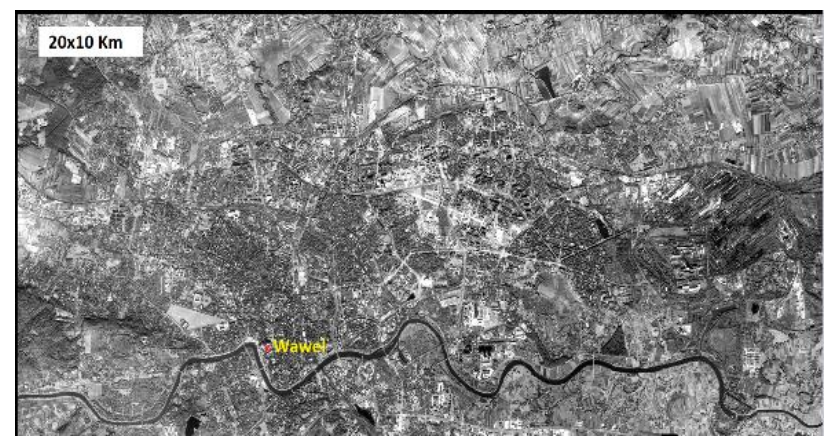

Figure 2.A WVII image with the study area of Cracow and the Wawel castle along the river Wisla

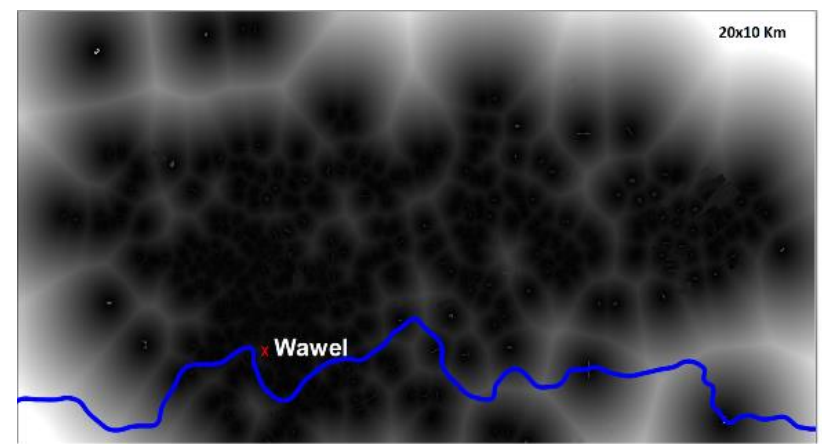

Figure 3 Visualization of the feature Distance to large buildings

Fig 3 displays the attribute "Distance to Building Volume Category $60 "$ in grey values. The Building category 60 is a selected subset of buildings with large volume derived from the 2006 Lidar image of Cracow. The River Wisla as a blue line draped over this to ease visual comparison. Fig 3 shows the distance of all 1,3 million objects towards their nearest object in the class Building 60. If the distance is very small, the value is low and the display is dark. Vice versa, when the distance to any building in category 60 is very large, the value is high and therefore bright. There are 541 large buildings in class 60 and 541 "Bubbles". These bubbles approach the characteristics of Thiessen polygons. 


\subsection{The repetitive patterns of city blocks}

Intuitive and empirical findings on urban mapping support the assumption that similar buildings are encountered as grouped in city blocks. At least for European conditions this is often the case. This hints at the fractal nature of European cities, where repetitive patterns can be observed within city blocks. Buildings within a certain category of „Building Volume” can then be expected to have several neighbours with similar values for „Building Volume”. Although this seems to be logic for planned city blocks, the characteristic of similarity for neighbouring buildings still can be observed among organic/historical growth patterns in European cities. It is now assumed that neighbourhoods with very large values for „Building Volume” cover the area of Dense Urban fabric. Vice versa, the buildings with small values for "Building Volume" are associated with sparse urban fabric. The latter form the use case for this study using the simplest parameters to prove the case. A visualization of all seven different distances to respective seven building categories can be combined in a colour composite (Fig4) to enhance the nested appearance of areas with similarities in distance to the same building category. Now these features must be translated to the protocol of the process tree.

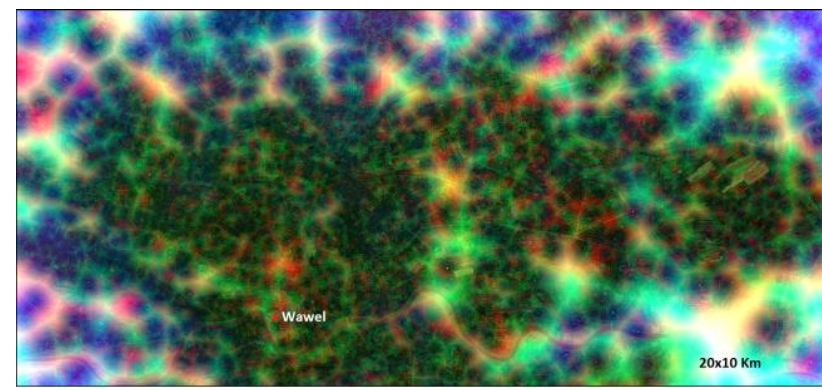

Figure 4 Visualization of the feature Distance to Buildings for 7 building classes. Each of the 1.3 million objects have 7 values for distance to building in 7 categories.

There are classes that can be calibrated better than large volume buildings and become the core classes in this introduction study to explain the central role of the feature distance to building class.

\section{EVALUATION}

\subsection{The Urban Atlas as reference}

The most recent version of urban atlas can now be used to Evaluate the classification in 7 classes.

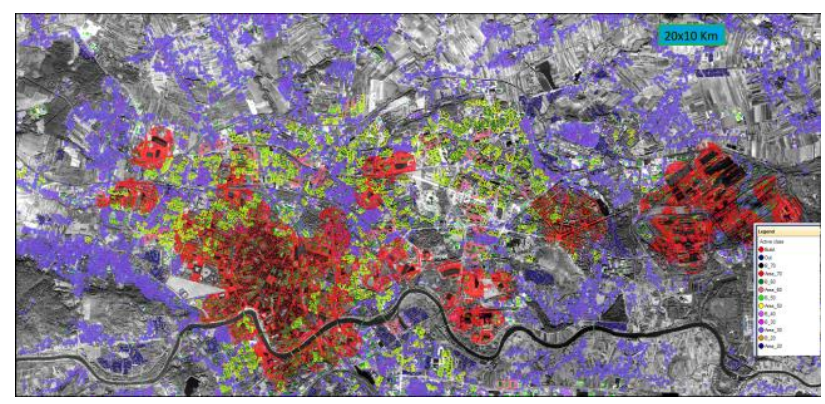

Figure 5 Visualization of the classification
To confront the classification results with a state of the art detailed product. The various classes must be split because not all urban areas are within the urban fabric. Most notably the class of Allotment gardens show a typical deviation from the other urban classes. The in-depth role will be described in the follow up of this extended abstract.

\section{CONCLUSIONS}

The classification of areas grouped by similar distance to building-type offers at the least a signal or flagging procedure to highlight urban atlas polygons that require an update.

\section{REFERENCES}

Avery, T.E., Gradon, L., 1992. Fundamentals of Remote Sensing and Airphoto Interpretation Berlin. 1992. PrenticeHall, Inc.

Bossard,M., Feranec,J., Otahel,J., 2000 CORINE Land Cover Technical Guide Addendum 2000, European Environment Agency Technical Report 40 http://www.eea.europa.eu/publications/tech40add

European Union, (2011). Mapping Guide for a European Urban Atlas. Available at http://www.eea.europa.eu/data-andmaps/data/urban-atlas.

Gangkofner, U., Weichselbaum, J., Kuntz, S., Brodsky, L. Larsson, K., de Pasquale, V., 2010 2010, Update of the European High-resolution Layer of Built-up Areas and Soil Sealing 2006 with Image2009 Data.Remote Sensing for Science, Education, and Natural and Cultural Heritage Rainer Reuter (Editor) EARSeL,

Lillesand, T.M, Kiefer, R.,Chipman J., 2015. Remote Sensing and Image Interpretation (7th edition).. John Wiley \& Sons,

Marr, D. (1982). Vision: A Computational Investigation into the Human Representation and Processing of Visual Information. San Francisco, CA: W. H.Freema

Montenegro, N., Duarte, J.P., 2009, CUMINCAD Computational Ontology of Urban Design: Towards a City Information Model , Computation: The New Realm of Architectural Design, Istanbul Technical University, Turkey, 